\title{
Transient multimodality in relaxation from an unstable state
}

\author{
Jan Iwaniszewski* \\ Institute of Physics, Nicholas Copernicus University, Grudzigdzka 5, 87-100 Toruń, Poland \\ P. V. E. McClintock ${ }^{\dagger}$ and N. D. Stein ${ }^{\ddagger}$ \\ School of Physics and Materials, Lancaster University, LA1 4 YB, United Kingdom
}

(Received 27 June 1994)

\begin{abstract}
We analyze a relaxation process from an unstable state during which transient multimodality occurs. This phenomenon is investigated experimentally on an electronic analog circuit which mimics an overdamped bistable oscillator driven by Gaussian white noise. The oscillator potential is a sixth-order polynomial $U(x)$. The measured times and positions at which new maxima appear in the probability distribution function agree well with the theoretical predictions. Although the initial stage of relaxation is governed by the noise the occurrence of transient multimodality is of the deterministic nature only. It is shown that the shape of the potential allows for the coexistence of three probability distribution peaks during a sizable interval of time, even though there is no long "flat" region in the potential where $U^{\prime}(x)$ is very small. Finally, the concept of marginality with reference to unsteady states is discussed.
\end{abstract}

PACS number(s): 05.40. $+\mathrm{j}, 02.50 .-\mathrm{r}$

\section{INTRODUCTION}

Consider a dynamical system subject to random noise. Its state may be characterized by the probability distribution function, in which the number of maxima is the dominant feature. As certain parameters associated with the system are altered, it evolves towards a new state having, perhaps, a different number of probability distribution peaks. If, for some period during the evolution, the number of probability distribution maxima is larger than in the initial and stationary states, one speaks about the phenomenon of transient multimodality (TM). It means, that for a sizable interval of time, the given system can be found with comparable probability in any one of a number of states that is larger than the number of its steady states. Such behavior was first reported by G. Nicolis and co-workers [1-3] in the theoretical study of explosive chemical reactions and combustion. Later on the possibility of the appearance of TM was mentioned for some optical bistable systems [4-7] and for a Brownian particle in shear flows [8]. The theoretical predictions were confirmed by means of numerical simulations $[1,2,4-6,9,10]$ and experiments on optical bistability [7,10-13], an electronic circuit [10], a laser with a saturable absorber [14], a semiconductor laser [15], electrohydrodynamic convection in nematic liquid crystals [16], combustion processes [17], and also through studies of the dynamics of error growth in numerical calculations [18].

The theoretical description associates TM with evolu-

\footnotetext{
*Electronic address: jiwanisz@phys.uni.torun.pl

${ }^{\dagger}$ Electronic address: pya007@cent1.lancs.ac.uk

${ }^{\ddagger}$ Electronic address: pya005@cent1.lancs.ac.uk
}

tion in a potential having a flat plateau, for which critical slowing down occurs $[4,6]$. The fluctuations driving the system accelerate the evolution of some stochastic realizations over the flat region, after which the system is rapidly switched to the vicinity of a stable state $x_{s}$. Since some stochastic realizations still remain at the plateau region one observes the "bunching" of trajectories in two places, namely at the plateau and in the bottom of the potential well. So the probability distribution possesses two maxima. This phenomenon arises some time after the initial probability distribution peak reaches the plateau and it disappears again once an appropriate mass of probability distribution (i.e., a sufficient proportion of stochastic trajectories) has left the flat part of the potential. Thus, there is a discontinuity in the evolution of the probability distribution maximum.

It was argued $[19,20]$ that such behavior can occur if the evolution of a system is characterized by two different time scales. The longer one results from the long induction stage at the potential plateau. It is of the order of the mean first passage time (MFPT) $T$ needed to reach the edge of the flat region. However, due to the essential role played by fluctuations at this stage of the evolution, the actual value of this time for a given realization is distributed within an interval of the order of the standard deviation $\Delta T$ of the MFPT. The shorter time scale $t_{d}$ is associated with the deterministic transit from the edge of the plateau to the bottom of the potential. In terms of these quantities the type of evolution can be characterized by the ratio $\eta=t_{d} / \Delta T$. TM is possible provided that $\Delta T$ is of the order of the long time scale $T$, i.e., $\eta<1$. In contrast, if $\eta>1$, most of the stochastic trajectories leave the plateau region before any of them reach the vicinity of $x_{s}$. Thus the probability of finding the system somewhere between the plateau and the bottom is large and, consequently, the proba- 
bility distribution peak travels continuously towards its stationary position. The relation $\eta<1$ is also known [21] to apply close to a marginal state $\left[U^{\prime}(x)=U^{\prime \prime}(x)=0\right]$ but not in the vicinity of an arbitrary unstable state $x_{u}\left[U^{\prime}\left(x_{u}\right)=0, U^{\prime \prime}\left(x_{u}\right)<0\right]$. Hence some authors $[6,14,20]$ conclude that, generally, TM is not expected during relaxation from the top of a potential barrier. The parabolic curvature of the barrier seems to be too steep to generate a distribution of $T$ wide enough that $\Delta T$ and $t_{d}$ are of different time scales. It should be noted, however, that this conclusion stemmed from the analysis of only one type of potential, namely the quartic potential

$$
U(x)=\frac{1}{4} b x^{4}+\frac{1}{2} a x^{2},
$$

(with $b>0, a<0$ ) that is usually considered while dealing with the decay of an unstable state (e.g., [22]).

Recently, however, one of the present authors [23] proved that TM can also arise during the evolution from an unstable state $x_{u}$. This effect applies to a very wide class of systems, even very far from marginality. It turns out that the type of evolution (with or without TM effect) to be anticipated depends only on the sign of the fourth derivative of the potential in the unstable state-if it is negative some new peaks must appear before the initial maximum at $x_{u}$ disappears. However, it also means that the potential curvature at the unstable state is much steeper than in the parabolic case, so that the system should leave the vicinity of $x_{u}$ very quickly. How can one reconcile this result with the previous discussion in terms of time scales? The main aim of the present paper is to answer this question.

We consider a one-dimensional overdamped oscillator subject to the force derived from a double-well potential and a Gaussian white noise. Its evolution is governed by the Langevin equation

$$
\dot{x}=-U^{\prime}(x)+\xi(t),
$$

with a symmetrical sixth-order polynomial potential

$$
U(x)=\frac{1}{6} c x^{6}+\frac{1}{4} b x^{4}+\frac{1}{2} a x^{2} .
$$

The parameter $a<0$ fulfills the requirement of instability at $x_{u}=0, b$ determines the sign of $U^{I V}(0)$ and $c>0$ ensures the existence of stable states $\pm x_{s}$. The noise $\xi(t)$, of zero mean, is characterized by its correlation function

$$
\left\langle\xi(t) \xi\left(t^{\prime}\right)\right\rangle=2 q \delta\left(t-t^{\prime}\right)
$$

where $q$ is the noise strength.

To confirm that TM really occurs during the decay of an unstable state we performed some electronic analog experiments. The experimental arrangements are described in Sec. II. The results are reported and compared with previously obtained [24] analytical formulas characterizing TM in Sec. III. The problem of the different time scales and the possibility of the appearance of TM are discussed in Sec. IV and some conclusions are drawn in Sec. V.

\section{ELECTRONIC ANALOG SIMULATIONS}

The utility of electronic analog techniques for modeling stochastic dynamics has been demonstrated in many contexts (see, e.g., $[25,26]$ ). An electronic circuit is in many ways the simplest "real physical system" in which a required theoretical problem can be investigated. The actual circuit used to model (2)-(4) is shown schematically in Fig. 1 (cf. [27]). It is based on three analog multipliers (Analog Devices, AD534) and a Miller integrator. Two independent random signals $\Xi$ and $Z$ are supplied from standard exponentially correlated Gaussian noise generators [28]. Since the noise correlation times are of the order of one tenth of the time constant of the Miller integrator, they are perceived as effectively white noises [26] with correlation functions

$$
\begin{gathered}
\left\langle\Xi(\tau) \Xi\left(\tau^{\prime}\right)\right\rangle=2 \theta_{\xi} V_{\xi}^{2} \delta\left(\tau-\tau^{\prime}\right), \\
\left\langle Z(\tau) Z\left(\tau^{\prime}\right)\right\rangle=2 \theta_{\zeta} V_{\zeta}^{2} \delta\left(\tau-\tau^{\prime}\right),
\end{gathered}
$$

where $V_{\xi}^{2}$ and $V_{\zeta}^{2}$ are the variances of the noise voltages [29].

A square-wave signal operates periodically a solid-state switch $S$ (DG303ACJ) to alter the system between two versions of the circuit. The first one (disconnected in Fig. 1) prepares the required distribution of initial conditions and it operates within a linear Langevin equation [29]

$$
\frac{d V_{x}}{d \tau}=-\lambda V_{x}+Z_{\zeta}(\tau)
$$

Since the half-period of the switching signal is much longer than the relaxation time of this circuit the stationary distribution of (7), namely a Gaussian distribution centered at $V_{x}=0$, is generated. During the second halfperiod the main circuit comes into action (as in Fig. 1) giving [29]

$$
\frac{d V_{x}}{d \tau}=-\gamma V_{x}^{5}-\beta V_{b} V_{x}^{3}-\left(\alpha_{1} V_{a}+\alpha_{2} V_{b}^{2}\right) V_{x}+\Xi(\tau)
$$

After the simplifying scaling $V_{x}=A x$ and $\tau=B t$

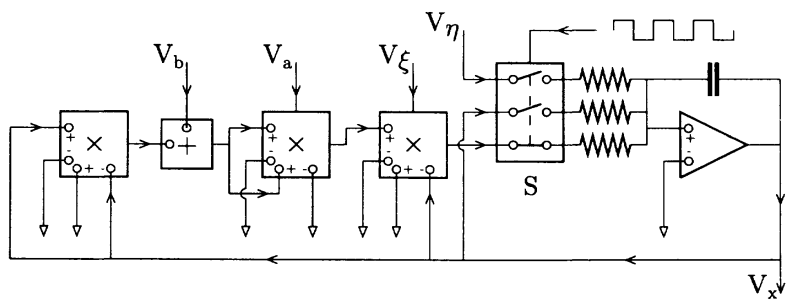

FIG. 1. Block diagram of the electronic circuit used to investigate the evolution of Eqs. (2)-(4) with randomly distributed initial conditions. The minus and plus signs inside the multipliers mean respectively that there is or is not a change of sign during the multiplication. The signals connected to the top of the multipliers are added to the results of the multiplication. 
(also determined by circuit values) one obtains from (5) and (8) the required Eqs. (2)-(4) while Eqs. (6) and (7) guarantee Gaussian initial distribution with a variance $\rho_{0}$. The four voltages $V_{a}, V_{b}, V_{\xi}$, and $V_{\zeta}$ are set externally to vary the parameters of the potential $U(x)$, the noise strength $q$, and the width $\rho_{0}$ of the initial distribution.

The output, a time-dependent voltage $V_{x}(t)$, was analyzed by means of a Nicolet 1080 computer system. For each realization of $x(t)$ an initial state was first prepared according to (7) with the required statistics (an appropriate value of $\rho_{0}$ ). Then, as the system was switched to the main circuit, the Nicolet analog-to-digital convertor was triggered and an input sweep was acquired: 1024 values of $V_{x}(t)$ were digitized and recorded at equal time intervals, with 12-bit precision. The sweep was analyzed by examining each 16 th value and incrementing the corresponding point of a 64-level distribution. The process then repeated, building up a set of 64 64-point distributions separated by equal intervals of time, and was continued until the statistics were adequate. It turned out that $2 \times 10^{4}$ realizations was a sufficient number.

\section{EXPERIMENTAL RESULTS}

The appearance of TM in (2)-(4) was confirmed immediately by the experimental results. An example of the time evolution of the probability distribution $W(x, t)$ for $b<0$ is exhibited as a three-dimensional plot in Fig. 2(a). The existence of a time regime within which three probability distribution peaks coexist is clearly evident. A comparison of the instantaneous experimental probability distribution with the same parameter values for several times in the stage of evolution when the new maxima appear is shown in Fig. 2(b). Notwithstanding the statistical fluctuations in the shape of $W(x, t)$, the trimodal character of the probability distribution at certain times is clearly demonstrated.

It follows from [24] that, for the potential (3), TM can appear not only for $b<0$ but also for some positive values of $b$, namely for $b<b_{c}=\sqrt{-\frac{5}{3} a c}$. Examples of the instantaneous probability distribution measured for positive $b$ at three times are given in Fig. 3. It is seen, that, within the experimental accuracy, the evidence of TM is very weak. Over a comparatively long period of time, the probability distribution remains very flat, almost uniform, and the difference between the heights of maxima and minima is very small, so that the effect of TM is rather insignificant. As the value of $b$ was reduced from $b_{c}$ the phenomenon of TM became more clearly resolved, although it lasted for a very short time. As $b$ crossed zero the coexistence of three probability distribution peaks became evident and the TM persisted for a very long time. Such behavior agrees well with the previous analysis [24] which distinguished short-life $\left(0<b<b_{c}\right)$ and long-life $(b<0)$ forms of TM. In the former case, the properties of the potential determine the finite time of coexistence of the three peaks, while in the latter case, it is only the strength of the noise that determines the time at which the middle peak disappears. Hence, if $q$ is very small, the effect will be observed over rather a long period and TM
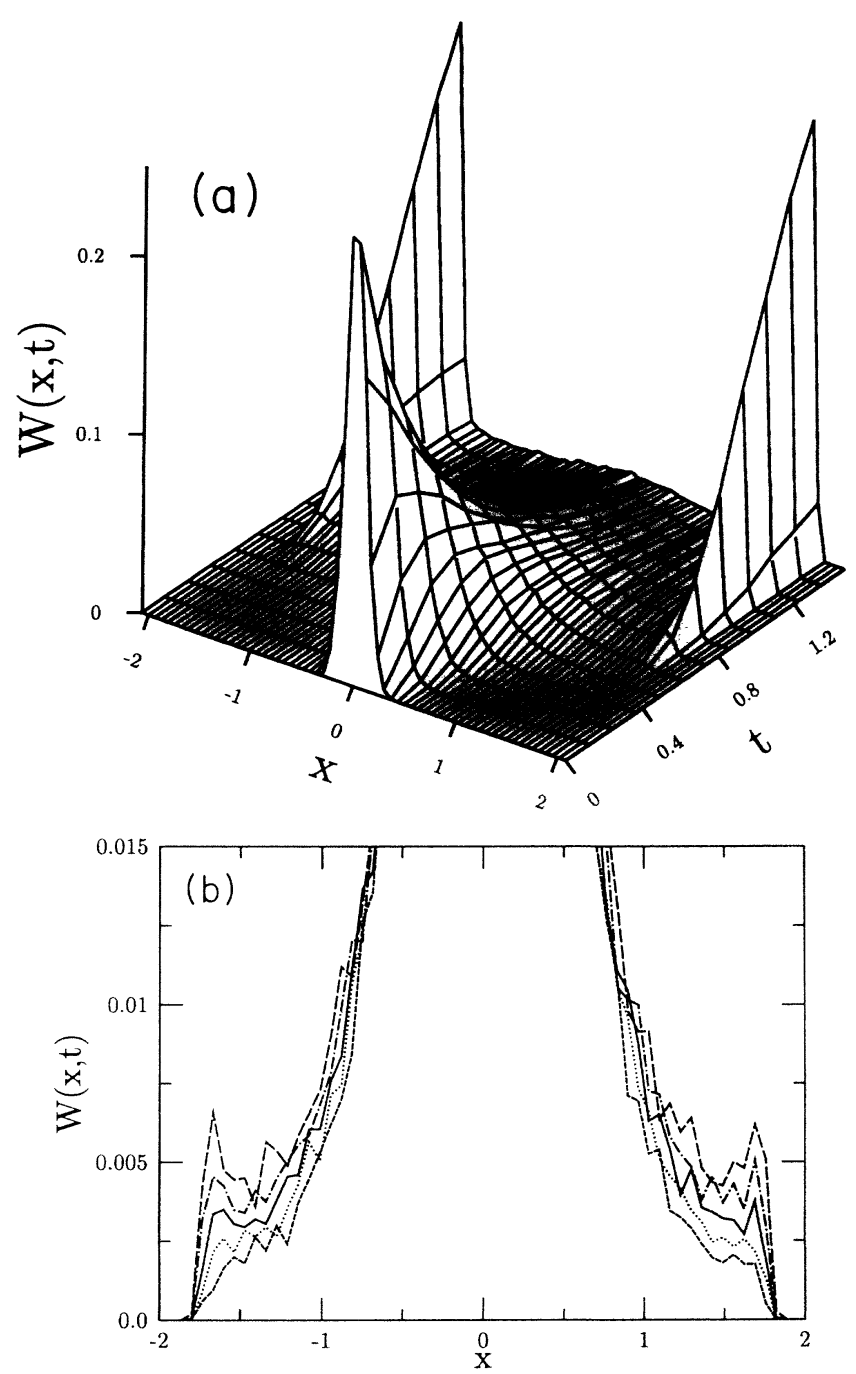

FIG. 2. Time evolution of the probability distribution $W(x, t)$ for $a=-2, b=-2.47, c=1, \rho_{0}=0.01$, and $q=0.02$ : (a) a three-dimensional plot and (b) a plot of the lower part of instantaneous probability distribution for $t=0.56$ (short-dashed line), 0.58 (dotted line), 0.60 (solid line), 0.63 (dot-dashed line), and 0.65 (long-dashed line).



FIG. 3. Cuts through the probability distribution $W(x, t)$ for $a=-2, b=0.99, c=1, \rho_{0}=0.01, q=0$, at times $t=0.93$ (dotted line), 0.99 (solid line), and 1.04 (dashed line). 
eventually seems to disappear because of the experimental indistinguishability of the middle maximum and the minima.

It was shown in [24] that both sources of randomness, i.e., the random initial conditions and the stochastic force affect the appearance of TM. The simpler case for theoretical treatment is that of deterministic evolution with randomly distributed initial states. In [24] some calculations for small $\rho_{0}$ were performed. In particular, the formulas for the critical time $t_{c r}$ and position $x_{c r}$ of the appearance of new maxima [i.e., values which satisfy $\left.W^{\prime}\left(x_{c r}, t_{c r}\right)=W^{\prime \prime}\left(x_{c r}, t_{c r}\right)=0\right]$ read

$$
\begin{aligned}
t_{c r}^{2} \approx & \frac{1}{2 a} \ln \left[\rho_{0}\left(-5 \frac{c}{a} x_{c r}^{2}-3 \frac{b}{a}\right)\right] \\
& +\frac{1}{2 a\left(x_{s}^{2}+p\right)}\left[x_{s}^{2} \ln \left(1+\frac{x_{c r}^{2}}{p}\right)\right. \\
& \left.+p \ln \left(1-\frac{x_{c r}^{2}}{x_{s}^{2}}\right)\right], \\
x_{c r}^{2} \approx & -\frac{13 b}{20 c}+\frac{7}{20} \sqrt{\left(\frac{b}{c}\right)^{2}-\frac{200 a}{49 c}}
\end{aligned}
$$

where the stable states $\pm x_{s}$ of the potential $U(x)$ are given as

$$
x_{s}^{2}=-\frac{b}{2 c}+\frac{1}{2 c} \sqrt{b^{2}-4 a c},
$$

and

$$
p=\frac{b}{2 c}+\frac{1}{2 c} \sqrt{b^{2}-4 a c} .
$$

These formulas, which approximate well the exact numerical data of [24], are compared with the present experimental data in Figs. 4 and 5 for several values of $\rho_{0}$ $(q=0)$. The dependence of $x_{c r}$ on the initial distribution width $\rho_{0}$ is small compared to the experimental inaccuracy, so only the experimental data for $\rho_{0}=0.01$ are shown on Fig. 4. Due to some slight asymmetries in the circuit the probability distribution is not exactly symmetric, and so the positions of left and right critical points are indicated separately; the averages of the left and right critical time values are shown by the data in Fig. 5.

The experimental fluctuations in the shape of $W(x, t)$ make it impossible to identify the exact time of appearance of a new maximum. To be sure that a given small hump is indeed a new probability distribution peak, and not just a fluctuation, the value of $t_{c r}$ is overestimated a little. Thus, the experimental data in Fig. 5 are slightly greater than the analytical ones. Similarly, since a new maximum tends almost immediately after its appearance to the vicinity of its stationary position (see Fig. 3 in [24]) the experimental values of $x_{c r}$ are a little higher than the theoretical ones. Another source of error, but one that we believe was unimportant, comes from the finite grid of voltage values - the whole range of voltages, which

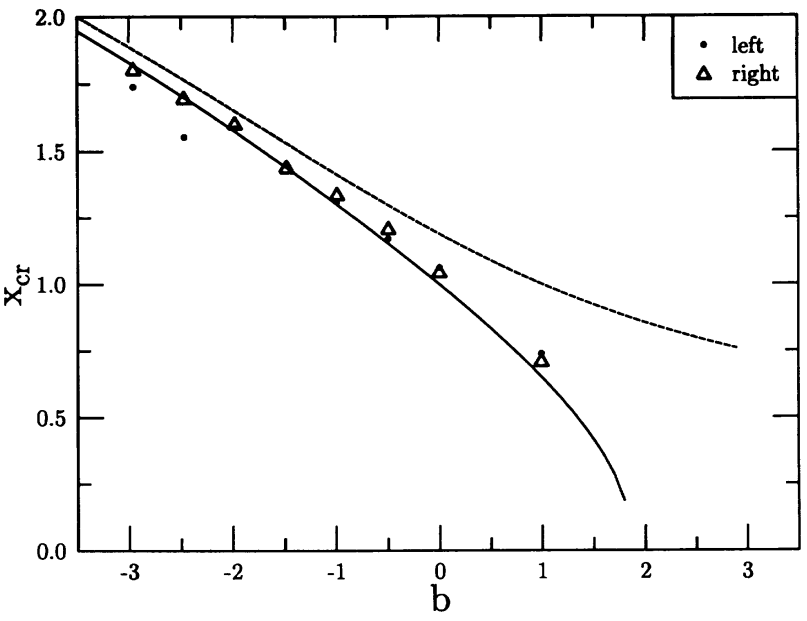

FIG. 4. The absolute value of the position $x_{c r}$ at which new extrema appear, plotted as a function of $b$ for $a=-2, c=1$, $\rho_{0}=0.01$, and $q=0$. The solid line represents the theory of Eq. (10) and the data points represent the experimental results for the extrema located to the left (dots) and to the right (triangles) with respect to the unstable point $x_{u}$. The position of the stationary maximum $x_{s}(11)$ is shown by the broken line.

was about $15-20 \%$ greater than the distance between the stationary maxima, was divided into 64 intervals. Despite the nonideality introduced by this coarse graining, remarkably good agreement is obtained between the theoretical and experimental results.

It was argued in [24] that, for $q \ll 1$ the formulas of deterministic evolution (9) and (10) may be used to describe the stochastic evolution if $\rho_{0}$ in (9) is replaced by $\rho_{\infty} \equiv \rho_{0}-q / a$. This is a consequence of the lownoise limit for which a stochastic solution may be ap-

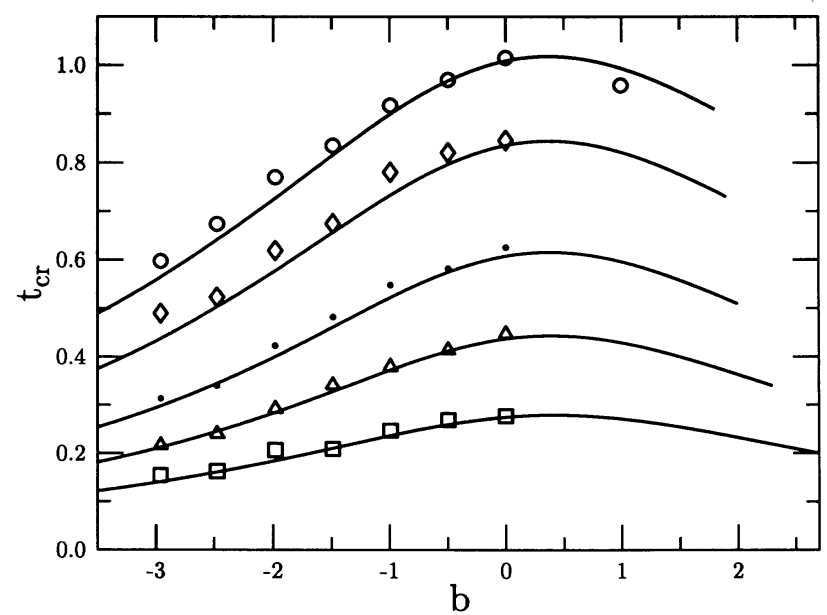

FIG. 5. The time $t_{c r}$ at which new extrema appear, plotted as a function of $b$ for $a=-2, c=1$, and $q=0$. The solid lines represent the theory of Eq. (9) and the data points represent the experimental results, taken as the average of the times at which the left and right maxima appear. The variance of the initial distribution, from top to bottom, is $\rho_{0}=0.01,0.02,0.05,0.10$, and 0.20 . 
proximated by a deterministic evolution with a time dependent "initial distribution" [30]

$$
W_{\text {in }}(x, t)=[2 \pi \rho(t)]^{-1 / 2} \exp \left[-x^{2} / 2 \rho(t)\right],
$$

where

$$
\rho(t)=\rho_{0}-\frac{q}{a}[1-\exp (2 a t)]
$$

Since, as follows from [24], $t_{c r} \sim|\ln q|$, the expression (14) for $\rho(t)$ may be approximated by its asymptotic value $\rho_{\infty}$. The comparison of experiment and theory for evolution exactly from the unstable state $x_{u}=0$ (within the experimental accuracy), i.e., with $\rho_{0}=0$, and with spread initial distribution with $\rho_{0}=0.01$ is shown in Figs. 6 and 7. As before, the position of $x_{c r}$ is reconstructed quite well by (10), but the consistency of theory and experiment for the critical time $t_{c r}$ is less good. There are a number of reasons for this discrepancy. First, at present, the experimental fluctuations of the shape of the probability density seem to be stronger due to the permanent operation of the stochastic force on the system, so that the overestimation of $t_{c r}$ is greater. The other reasons are of a theoretical character. The use of formula (9) to approximate $t_{c r}$ using $\rho_{\infty}$ instead of $\rho_{0}$ assumes that, during the whole time interval $\left[0, t_{c r}\right]$ the stochastic evolution takes place retaining the fixed width of the "initial distribution." In fact, it depends on time and it changes during this interval from $\rho(0)=\rho_{0}$ to $\rho\left(t_{c r}\right) \neq \rho_{\infty}$ and $\rho_{0}, \rho\left(t_{c r}\right)<\rho_{\infty}[$ cf. (14)]. Consequently, the actual value of $t_{c r}$ shifts towards the values computed from (9) with smaller values of $\rho_{0}$. Finally, the formula (9) is itself an approximate one [24]. This point will be discussed further in the final section below.

\section{SHAPE OF POTENTIAL AND TIME SCALES}

Let us return now to the discussion of Sec. I on the relationship between the shape of the potential, time scales, and the possibility of TM occurring during the relaxation from an unstable state. The sufficient condition for TM

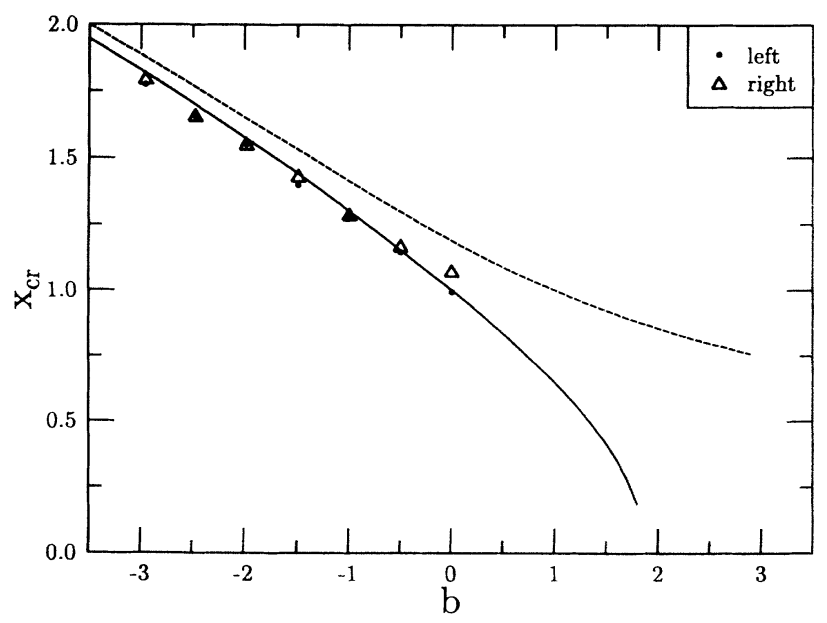

FIG. 6. As in Fig. 4 but for $\rho_{0}=0$ and $q=0.02$.

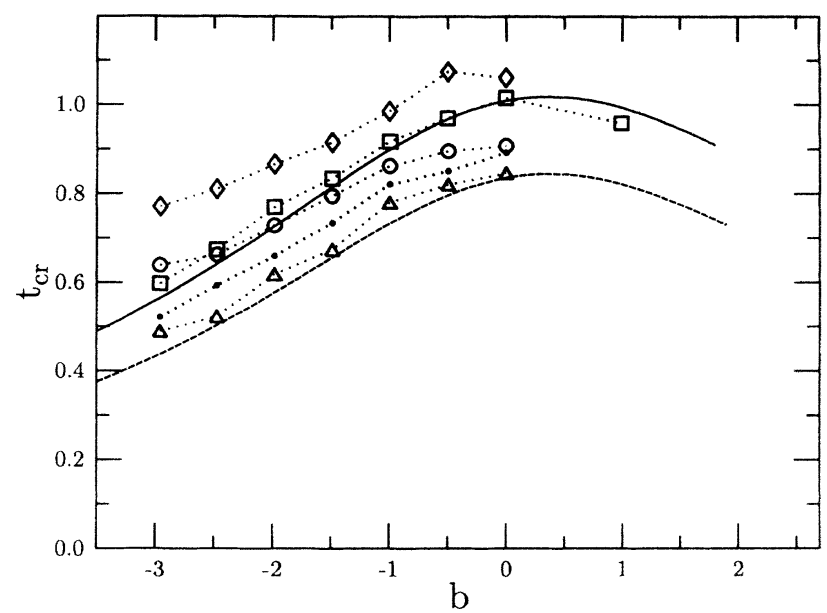

FIG. 7. As in Fig. 5 but with experimental results taken for different values of the pair of parameters $\rho_{0}$ and $q$, namely $(0.01,0.00)$ (squares), $(0.02,0.00)$ (triangles), $(0.00,0.02)$ (diamonds), $(0.00,0.04)$ (circles), and $(0.01,0.02)$ (dots). The corresponding values of $\rho_{\infty}$ are $0.01,0.02,0.01,0.02$, and 0.02 , respectively. The lines represent the theory of Eq. (9) for $\rho_{0}=0.01$ (solid) and 0.02 (broken). The dotted lines are guides to the eye.

in this case, i.e., $U^{I V}\left(x_{u}\right)<0$ means that the deterministic motion is accelerated as compared to the linear evolution [23], namely

$$
\dot{x}=-U^{\prime}(x) \approx|a|\left(1-\frac{b}{|a|} x^{2}\right) x .
$$

This is in contrast to the case of a quartic potential (1) for which the parameter $b$ in (15) is positive, so that it slows down the deterministic movement. Due to the acceleration that occurs when $b<0$ a stochastic trajectory, which is just thrown out from the unstable state and outside the probability distribution peak, leaves the vicinity of $x_{u}$ and reaches the bottom of the potential well before the initial distribution peak has emptied. In other words, although the evolution near $x_{u}$ develops in a much shorter time than the evolution across a flat plateau or near a marginal point, nevertheless the transit regime towards the bottom of the potential also occurs much faster. Consequently, since both stages of evolution (i.e., both time scales) are accelerated, the ratio $\eta$ may still be small enough to allow the manifestation of TM.

To reveal the differences between the relaxations for the potentials (3) and (1) more clearly one must scale the potentials to make them as similar as possible. It is obvious that the whole process of evolution depends not only on the shape of the potential in the neighborhood of $x_{u}$, but also on the distance between $x_{u}$ and $x_{s}$, and on the difference between the potentials at these points. Hence, to avoid any dependence on these specific properties of the potential wells, we scale both systems in such a way that the potential minima are set at $y_{s}= \pm 1$ and the height of the potential barrier is equal to 1 . Thus we obtain

$$
\widetilde{U_{4}}(y)=4\left(\frac{1}{4} y^{4}-\frac{1}{2} y^{2}\right)
$$


and

$$
\widetilde{U_{6}}(y)=\frac{12}{1+3 p}\left[\frac{1}{6} y^{6}+\frac{1}{4}(p-1) y^{4}-\frac{1}{2} p y^{2}\right],
$$

where $0<p<\infty$ guarantees a double-well shape for the potential. As $p$ reaches its boundary values $\widetilde{U_{6}}(y)$ takes the following forms:

$$
\begin{aligned}
p \rightarrow 0 & \widetilde{U_{6}}(y) & \rightarrow 12\left(\frac{1}{6} y^{6}-\frac{1}{4} y^{4}\right) \\
p \rightarrow+\infty & \widetilde{U_{6}}(y) & \rightarrow \widetilde{U_{4}}(y) .
\end{aligned}
$$

The potential $\widetilde{U_{6}}(y)$ given by $(17)$ is just an intermediate one between the marginal (18) and quartic (16) ones. The former is a generic potential at a subcritical pitchfork bifurcation point [31] and the latter is a generic form for a supercritical pitchfork bifurcation [22]. Figure 8 exhibits a plot of the potential (17) for various $p$ values. It is seen that in the vicinity of the unstable point for any $p<+\infty$ the potential $\widetilde{U_{6}}(y)$ is flatter than $\widetilde{U_{4}}(y)$ which is, at first sight, a quite unexpected property (compare the discussion in Sec. I).

However, the character of the evolution within this flatpotential area is quite different from that near a marginal state. In this last case, the flatness of the potential is determined by the relation to the noise, namely $U^{\prime \prime}\left(x_{m}\right)$ is of the order of $\sqrt{q}[32]$. Such a shape leads to critical slowing down and this is just the case described in Sec. I. In contrast, the flatness in Fig. 8 stems from the comparison with other parts of the potential. The adjacent parts of the potential are much steeper than in the vicinity of an unstable point $x_{u}$, which obviously cannot lead to critical slowing down.

Now let us estimate the time scales of the problem. Colet et al. [20] have investigated the relaxation dynamics from the situation at the bifurcation point of a subcritical pitchfork bifurcation $[a=0, b<0$ in (3)] to a situation well above the bifurcation point $(a \ll 0)$. They have shown that the early and intermediate regimes of relaxation may be described by the single parameter

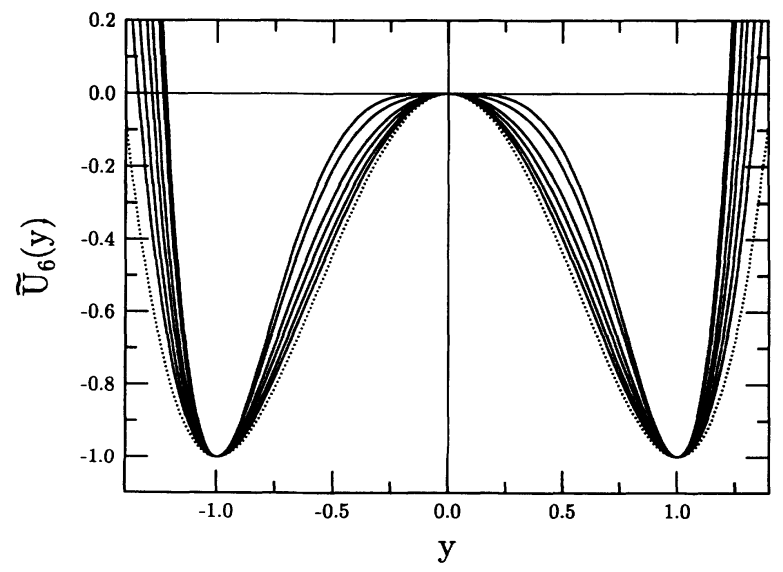

FIG. 8. Scaled potentials $\widetilde{U_{4}}(y)$ (16) (dotted line) and $\widetilde{U_{6}}(y)(17)$ for $p=0,0.1,0.4,1$, and 2 (solid lines). The curve with $p=0$ is the flattest curve at the potential barrier.

$$
k=\frac{|a|}{\sqrt{q|b|}},
$$

This quantity determines the relative importance of the linear term in comparison with the nonlinear one and the noise intensity, and hence measures the distance from marginality $(a=k=0)$. In the small $q$ approximation the lifetime of the state $x_{u}$, identified with the MFPT of a threshold $x_{t h}$ outside the vicinity of $x_{u}$, in the two limiting cases reads

$$
T=\frac{1}{16} \sqrt{\frac{2}{q|b|}} \Gamma\left(\frac{1}{4}\right)^{2}
$$

for the marginal case $(k=0)$, and

$$
T=\frac{1}{2|a|} \ln \frac{|a| x_{t h}^{2}}{q}
$$

well above the bifurcation point $(k \gg 0)$, i.e., for a relaxation from an unstable state. Although the functional dependence of $T$ on $q$ in both these formulas is disparate, $q$ dependence identifies the mean MFPT as a long-time scale. A quite different situation concerns the standard deviation of the MFPT. For the marginal case $(k=0)$ it depends on $q$, i.e.,

$$
\Delta T=\sqrt{\frac{0.5520}{q|b|}}
$$

so it is also of the long-time scale, while for the unstable case $(k \gg 0)$ its leading term does not depend on $q$, namely

$$
\Delta T=\frac{\sqrt{\Psi^{\prime}(0.5)}}{2|a|}
$$

where $\Psi$ is the digamma function. Hence it is not of the same time scale as $T$. This led Colet et al. [20] to suggest that TM could not arise for the decay of an unstable state.

In order to estimate the second time scale of the transition from the vicinity of $x_{u}$ towards $x_{s}$, one may approximate the steep part of $U(x)$, i.e., the section lying within the transit region between $x_{u}$ and $x_{s}$, by the tangent at the inflection point of the potential. Thus we have

$$
t_{d}=\frac{\left|U\left(x_{s}\right)-U\left(x_{u}\right)\right|}{U^{\prime}\left(x_{i}\right)^{2}},
$$

where the dependence on the slope of the potential in its steep part is obvious. In Table I we make a comparison of $\Delta T$ and $t_{d}$ for the scaled potential $\widetilde{U_{6}}(17)$ for $q=0.01$ and a few values of $p$. Evidently, for the marginal case $(p=0)$ the ratio $\eta$ is much lower than one and, according to the discussion in Sec. I and Refs. $[19,20]$ TM is a natural consequence of this. For the remaining two cases $\eta$ is of order unity. From the theory [23] and the experiment (Sec. III) it follows that for $p=1$, TM exists, while for $p \rightarrow \infty$ it does not. This means, that if the time scales of the problem are of the same order TM may or may not occur. The decisive role in determining how the system will evolve is performed by the order of the nonparabolic, saturation term in the potential. Namely, if it is of the 
TABLE I. Variance of the MFPT $\Delta T$, the transit time $t_{d}$ and their ratio $\eta$ for $q=0.01$ and three values of the parameter $p$ which determines the shape of the potential $\widetilde{U_{6}}$ (17). The corresponding values of the parameters $a, b, c$, and $k$ are also given

\begin{tabular}{cccccccc}
\hline \hline$p$ & $a$ & $b$ & $c$ & $k$ & $\Delta T$ & $t_{d}$ & $\eta$ \\
\hline 0 & 0 & -12 & 12 & 0 & 2.14 & 0.20 & 0.09 \\
1 & -3 & 0 & 3 & $\infty$ & 0.37 & 0.39 & 1.05 \\
$\infty$ & -4 & 4 & 0 & 20 & 0.28 & 0.42 & 1.52 \\
\hline \hline
\end{tabular}

sixth order $(c>0, b=0)$ TM appears whereas, if it is of the fourth order $(c=0, b>0)$ the relaxation occurs in the usual way, without TM. Moreover, if both the fourthand sixth-order terms exist $(b \neq 0, c>0)$ the kind of relaxation depends on the relation between them, that is, TM appears for $b<b_{c}$. Although for $0<b<b_{c}$ the manifestation of TM is very weakly displayed, nevertheless for any $b<0$ this phenomenon is very pronounced. Hence, if $b$ is equal to zero or if it is slightly negative so that the parameter $k$ is very large, TM will appear. This shows that the conclusion of [20] does not apply to the potential (3) considered here. Colet et al. [20] attribute the appearance of TM to the dependence of $\Delta T$ on the noise strength similar to the relaxation within a flat region of a potential. Here, we argue that $\Delta T$ need not depend on noise at all. An appropriate shape of potential automatically guarantees that TM will arise.

\section{CONCLUSIONS}

The electronic analog experiments have proven that the occurrence of TM during the relaxation from an unstable state is a genuine phenomenon that can occur in real physical systems. We were able to measure a time moment and a place for the appearance of some new maxima, and the existence of the multipeak probability distribution was observed over a sizable interval of time. This phenomenon manifests itself even very far from a marginal case, i.e., for $k \gg 0$. Thus the prediction in [23] about the necessity of relaxation with a discontinuous evolution of probability distribution maximum for $U^{I V}\left(x_{u}\right)<0$ is consistent with the experimental evidence. However, the short-life TM reported in [24] for $0<b<b_{c}$ is probably better considered as a stage of evolution with a very wide probability distribution, and not really as a multipeaked case. During this stage of evolution a system may be found with almost the same probability anywhere between the unstable $x_{u}$ and stable $x_{s}$ states. The genuine TM implies, on the other hand, that a system exists with a reasonable probability in any one of only a few well-defined states, i.e., near $x_{u}$ or near $\pm x_{s}$. Since the probability of being between $x_{u}$ and $x_{s}$ is very small one may speak about a rapid switching between the unstable and stable states. The switching process begins at a time $t_{c r}$ approximated by the formula (9). The experimental results show that (9) is quite good far from marginality but that it grows less accurate as one approaches marginality. This is a consequence of the inapplicability of the linear approximation at a marginal state which was used in the derivation of
(9) (cf. [30]). On the other hand, however, the applicability of this approximation far from the marginality comes from the independence of the relaxation process on the fluctuations. The random force is responsible only for the beginning of the evolution, while the whole character of the subsequent relaxation is governed by the deterministic factors [33].

Considering the two different time scales, which are distinguishable during the evolution, one must notice that their nature is quite different. The short time scale associated with the transition between $x_{u}$ and $x_{s}$ is strictly deterministic. The long one associated with a random triggering from $x_{u}$ depends on stochastic factors. Nevertheless, a dispersion associated with this stochastic stage of evolution is of a deterministic nature. Thus the ratio $\eta$ is a nonstochastic quantity, too, and hence it is obvious that the appearance of TM during a decay of an unstable state is a noise independent phenomenon [23]. In such a case, $\eta$ is not a good parameter for characterizing TM.

As mentioned in Sec. I some authors regard TM as a signature of marginality. Since, as we have shown, its occurrence is also possible very far from a marginal point, the concept of marginality should be revised. Usually one deals with a marginal equilibrium state, i.e., a state $x_{m}$ for which $U^{\prime}\left(x_{m}\right)=U^{\prime \prime}\left(x_{m}\right)=0$. Such a definition comes from the bifurcation (critical) behavior of a given system - the term marginality is associated with a steady state analysis. In contrast, TM is a time dependent phenomenon associated with unsteady states, which are not treated by a bifurcation theory.

On the other hand, bifurcation theory deals with only deterministic systems. It is well known [34], that the addition of fluctuations to a deterministic system may change radically its possible steady states - one speaks about noise-induced transitions. Transient bimodality occurring during the evolution across the plateau region of the potential is an analogous noise-induced phenomenon relative to unsteady states. The situation described in the present paper is noise independent, so it is an evolutionary counterpart of the deterministic bifurcation theory. Within the latter, a marginal state is a boundary state between two regions with different steady states. The region where TM occurs lies between the regions with the number of time dependent probability distribution peaks determined by the number of steady states. Thus, in analogy to the bifurcation theory, we may call this region a marginal one. Provided that the concept of marginality is generalized in this way, one may still speak of TM as a signature of the marginality of a system but taking it as an intermediate case between two regions where the probability distribution maxima develop continuously.

\section{ACKNOWLEDGMENTS}

One of us (J.I.) is very grateful for extremely warm hospitality during his stay at Lancaster University. This work was partly supported by the European Science Exchange Program (the Royal Society of London) and the Science and Engineering Research Council (UK). 
[1] F. Baras, G. Nicolis, M. Malek Mansour, and J. W. Turner, J. Stat. Phys. 32, 1 (1983).

[2] M. Frankowicz and G. Nicolis, J. Stat. Phys. 33, 595 (1983).

[3] M. Frankowicz, M. Malek Mansour, and G. Nicolis, Physica $125 \mathrm{~A}, 237$ (1984).

[4] G. Broggi and L. A. Lugiato, Phys. Rev. A 29, 2949 (1984).

[5] G. Broggi and L. A. Lugiato, Philos. Trans. R. Soc. London, Ser. A 313, 425 (1984).

[6] G. Broggi, L. A. Lugiato, and A. Colombo, Phys. Rev. A 32, 2803 (1985).

[7] L. A. Lugiato, G. Broggi, and A. Colombo, in Frontiers in Quantum Optics, edited by E. R. Pike and S. Sarkar (Hilger, Bristol, 1986), p. 231.

[8] H. S. Wio and D. H. Zanette, Phys. Rev. E 47, 384 (1993).

[9] F. De Pasquale and A. Mecozzi, Phys. Rev. A 31, 2454 (1985).

[10] F. Mitschke, R. Deserno, J. Mlynek, and W. Lange, IEEE J. Quantum Electron. QE-21, 1435 (1985).

[11] W. Lange, F. Mitschke, R. Deserno, and J. Mlynek, Phys. Rev. A 32, 1271 (1985).

[12] W. Lange, in Instabilities and Chaos in Quantum Optics II, edited by N. B. Abraham, F. T. Arecchi, and L. A. Lugiato, Vol. 177 of NATO Advanced Study Institute, Series B: Physics (Plenum, New York, 1988), p. 265.

[13] J. Nalik, W. Lange, and F. Mitschke, Appl. Phys. B 49, 191 (1989).

[14] E. Arimondo, D. Dangoisse, and L. Fronzoni, Europhys. Lett. 4, 287 (1987).

[15] P. Spano, A. Mecozzi, and A. Sapia, Phys. Rev. Lett. 64, 3003 (1990).

[16] S. Kai, M. Andoh, and S. Yamaguchi, Phys. Rev. A 46, R7375 (1992).
[17] A. Lemarchand, R. I. Ben Aim, and G. Nicolis, Chem. Phys. Lett. 162, 92 (1989).

[18] C. Nicolis and G. Nicolis, Phys. Rev. A 43, 5720 (1991).

[19] A. Valle, L. Pesquera, and M. A. Rodriguez, Opt. Commun. 79, 156 (1990).

[20] P. Colet, F. De Pasquale, and M. San Miguel, Phys. Rev. A 43, 5296 (1991).

[21] B. Caroli, C. Caroli, and B. Roulet, Physica A 101, 581 (1980).

[22] M. Suzuki, Prog. Theor. Phys. 56, 77 (1976).

[23] J. Iwaniszewski, Phys. Rev. A 45, 8436 (1992).

[24] J. Iwaniszewski, Phys. Lett. A 171, 278 (1992).

[25] L. Fronzoni, in Noise in Nonlinear Dynamical Systems, edited by F. Moss and P. V. E. McClintock (Cambridge University Press, Cambridge, 1989), Vol. 3, p. 222.

[26] P. V. E. McClintock and F. Moss, in Noise in Nonlinear Dynamical Systems (Ref. 25), p. 243.

[27] J. Casademunt, J. I. Jiménez-Aquino, J. M. Sancho, C. J. Lambert, R. Mannella, P. Martano, P. V. E. McClintock, and N. G. Stocks, Phys. Rev. A 40, 5915 (1989).

[28] N. G. Stocks, R. Mannella, and P. V. E. McClintock, J. Stat. Phys. 54, 1383 (1989).

[29] The parameters $\theta_{\xi}$ in (5), $\theta_{\zeta}$ in (6), $\lambda$ in (7), $\alpha_{1}, \alpha_{2}$, $\beta$, and $\gamma$ in (8) can be expressed in terms of component values in the electronic system (cf. $[25,26])$.

[30] J. Iwaniszewski, J. Phys. A 22, L337 (1989).

[31] P. Colet, F. De Pasquale, M. O. Cáceres, and M. San Miguel, Phys. Rev. A 41, 1901 (1990).

[32] H. Dekker and N. G. van Kampen, Phys. Lett. 76A, 101 (1980).

[33] F. T. Arecchi, A. Politi, and L. Ulivi, Nuovo Cimento B 71, 119 (1982).

[34] W. Horsthemke and R. Lefever, Noise-Induced Transitions (Springer, Berlin, 1984). 